\title{
Investigation of magnetomechanothermodiffusive processes in ferromagnetic body at given diffusing substance concentration on the surface
}

\author{
Solodyak M. \\ Pidstrygach Institute for Applied Problems of Mechanics and Mathematics of NASU \\ 3B Naukova str., 79060, Lviv, Ukraine
}

(Received 15 March 2015)

\begin{abstract}
The input equations describing the electromagnetic field interaction with the substance are suggested. By means of the methods of the similarity and dimensional theory, the system of equations of magnetomechanothermodiffusion and mechanics is reduced to a dimensionless form. Quantitative analysis of dimensionless criteria for the case of hydrogen diffusion in ferrum is carried out for three different meanings of initial temperature. The phenomena, for which dimensionless parameters are much less than a unit are not considered. A reduced coupled system of equations of the model for determination the concentration and temperature is written.
\end{abstract}

Keywords: electromagnetic field, magnetomechanothermodiffusion, ferromagnetic body, concentration of diffusing substances, methods of similarity and dimensional theory

2000 MSC: $58 \mathrm{~J} 65$

UDC: $539.3: 537.22$

\section{Introduction}

Mathematical models of magnetomechanothermodiffusion: macroscopic electrodynamics of moving media [1, 2], magnetothermoelasticity of ferromagnetic bodies [3], mechanothermodiffusion in multicomponent solid solutions $[4,5]$ etc. are cumbersome and unsuitable for practical investigation. The equations of these models contain a number of terms, which when considering these specific processes make little contribution when compared with other. Depending on the type of external action of the test material, separate terms of the system of equations can be neglected, i.e. to simplify the model essentially. For example, in $[6,7]$ the mathematical models that describe the processes of magnetothermodiffusion in ferromagnetic (both electroconducting and nonelectroconducting) bodies under the influence of an external time-periodic magnetic field are reduced to a system of equations of magnetothermomechanics.

In the present work the same procedure for ferromagnetic body, which is influenced by diffusing substance, the concentration $c_{1}$ of which is given on the body surface $\Sigma$ is carriedout:

$$
\left.c\left(\mathbf{r}_{0}, t\right)\right|_{\Sigma}=c_{1}
$$

where $\mathbf{r}_{0}$ is the radius-vector of the considered surface point, $t$ is time.

\section{The initial system of equations of magnetothermomechanodiffusion}

Perform the analysis of the mathematical model describing the relationship of electromagnetic, thermal, mechanical and diffusion processes. To do this, write down the basic equations of magnetothermomechanodiffusion. Let's start with the basic formulas of electrodynamics of slowly moving media. Thus, Maxwell's equation, the law of conservation of electric charge and material relations of electro- 
dynamics for ferromagnetic bodies are [3, 8]

$$
\begin{gathered}
\nabla \times \mathbf{H}=\dot{\mathbf{D}}+\mathbf{j}, \quad \nabla \times \mathbf{E}=-\dot{\mathbf{B}}, \quad \nabla \cdot \mathbf{B}=0, \quad \nabla \cdot \mathbf{D}=\rho q, \quad \rho \frac{d q}{d t}+\nabla \cdot \mathbf{J}=0 . \\
\mathbf{B}=\mathbf{B}(\mathbf{H}-\mathbf{v} \times \mathbf{D}), \quad \mathbf{D}=\varepsilon \varepsilon_{0}(\mathbf{E}+\mathbf{v} \times \mathbf{B}) .
\end{gathered}
$$

Here $\mathbf{E}$ and $\mathbf{H}$ are the vectors of stresses and $\mathbf{D}$ and $\mathbf{B}$ are the vectors of induction of electric and magnetic fields; $\mathbf{j}$ and $\mathbf{J}=\mathbf{j}-\rho q \mathbf{v}$ are the densities of total electric current and conduction current; $d / d t=\partial / \partial t+\mathbf{v} \cdot \nabla$ is the total time derivative; $\mathbf{u}$ and $\mathbf{v}=d \mathbf{u} / d t$ are the vectors of displacement and velocity of the center of mass of the body element; $\rho$ is the mass density; $q$ is the specific electric charge. The symbols " $\nabla \times$ " and " $\nabla$." mark the rotor and divergence operations, and the dot over the value is the partial time derivative; $\varepsilon$ and $\mu$ are the relative dielectric and magnetic permeability of the medium; $\varepsilon_{0}$ and $\mu_{0}$ are the electric and magnetic constant.

Balance relations for the mass density $\rho$, concentration $c$, momentum $\rho \mathbf{v}$, and the definition of deformation tensor $\hat{\mathbf{e}}$ according to Cauchy are

$$
\begin{gathered}
\frac{d \rho}{d t}+\rho \nabla \cdot \mathbf{v}=0, \quad \rho \frac{d c}{d t}+\nabla \cdot \mathbf{J}_{c}=0, \quad \hat{\mathbf{e}}=(\nabla \mathbf{u}+\mathbf{u} \nabla+\nabla \mathbf{u} \cdot \mathbf{u} \nabla) / 2, \\
\rho \frac{d \mathbf{v}}{d t}=\nabla \cdot \hat{\boldsymbol{\sigma}}+\rho q \mathbf{E}+\mathbf{j} \times \mathbf{B}+\mathbf{F} .
\end{gathered}
$$

Here $\hat{\boldsymbol{\sigma}}$ is the stress tensor, $\mathbf{J}_{c}$ is the concentration flow where

$$
\mathbf{F}=\frac{1}{2}\left(\frac{B(H)}{H}-\mu_{0} \mu(H)\right) \nabla\left(\mathbf{H}^{2}\right)
$$

is a part of ponderomotive force resulting due to magnetization.

Based on the general relations of Onsager theory [9] and the specific results of the kinetic theory for the case of weak interaction of diffusant with the skeleton [10], we write the expressions for the thermal $\mathbf{J}_{Q}$, diffusion $\mathbf{J}_{c}$ flows and conduction current density $\mathbf{J}$ :

$$
\begin{gathered}
\mathbf{J}_{Q}=-\kappa \nabla T+\frac{\kappa T_{0}}{\varphi_{1}}(\mathbf{E}+\mathbf{v} \times \mathbf{B})-\frac{2 \kappa T_{0}}{3 c_{n}}\left[\nabla c-\frac{\left(1-2 \nu_{p}\right)}{E_{p}} c_{n} \nabla \sigma\right]+\kappa_{l} \mu_{q} \nabla q, \\
\mathbf{J}_{c}=-\rho_{0} D\left(\nabla c+\frac{c}{2 T} \nabla T-\frac{\mu_{\sigma}}{\mu_{c}} \nabla \sigma+\frac{\mu_{q}}{\mu_{c}} \nabla q\right), \quad \mathbf{J} \equiv \lambda \mathbf{E}_{s} .
\end{gathered}
$$

Here

$$
\mathbf{E}_{s}=\mathbf{E}+\mathbf{v} \times \mathbf{B}-\frac{2 \varphi_{1}}{3 c_{n}}\left(\nabla c-3 c_{n} \alpha_{T} \nabla T-\frac{1-2 \nu_{p}}{E_{p}} c_{n} \nabla \sigma\right) .
$$

All definitions and notations in formulas (6)-(8) in the sequel, are such as in $[6,7]$.

Write the generalized Hooke's law

$$
\hat{\boldsymbol{\sigma}}=\frac{E_{p}}{1-2 \nu_{p}}\left[\frac{\nu_{p}}{1+\nu_{p}} e-\alpha_{T}\left(T-T_{0}\right)+\frac{\beta_{1}}{3} c\right] \hat{I}+\frac{E_{p}}{1+\nu_{p}} \hat{\mathbf{e}}-\hat{\boldsymbol{\pi}}
$$

and the heat conduction equations

$$
\frac{\rho c_{\sigma}}{\kappa} \frac{d T}{d t}=\Delta T+\frac{1}{\kappa}\left(Q-\rho T \frac{d s^{\prime}}{d t}\right)
$$

where

$$
Q=-\nabla \cdot \mathbf{I}_{Q}^{\prime}+\lambda \mathbf{E}_{s}^{2}-\mu_{c} \mathbf{I}_{c} \cdot \nabla\left(c-\frac{\mu_{T}}{\mu_{c}} T-\frac{\mu_{\sigma}}{\mu_{c}} \sigma\right)-\mathbf{v} \times \mathbf{F}
$$


are the total heat releases and

$$
s^{\prime}=\frac{\alpha_{T}}{\rho_{0}} \sigma+\gamma q+d_{1} c, \quad \mathbf{J}_{Q}^{\prime}=\mathbf{J}_{Q}+\kappa \nabla T
$$

is a part of entropy and heat flux not directly related to the thermal process [3].

To the system of equations of magnetothermomechanodiffusion (1)-(12) it is necessary to add the relevant thermal, mechanical and diffusion, initial, boundary and contact conditions corresponding to the particular case.

\section{The system of equations of magnetothermomechanodiffusion in dimensionless form}

The aim is to simplify the system (2)-(12) under the external action (1). Therefore, just as in [6, 7], using the methods of the theory of dimensions and similarity [11, 12], we introduce the corresponding characteristic values for the magnitudes under consideration and we reduce the system of equations (1)-(12) to a dimensionless form. All dimensionless values are denoted by a subscript "** (except time $\tau=t / t_{1}$, where $t_{1}$ is a characteristic diffusion time).

Let $l$ be a typical body size. Then for the dimensionless radius-vector and operators $\nabla, \Delta$, and the time operator $\partial / \partial t$ as well we will have

$$
\mathbf{r}_{*}=\mathbf{r} / l, \quad \nabla=\nabla_{*} / l, \quad \Delta=\Delta_{*} / l^{2}, \quad \partial / \partial t=t_{1}^{-1} \partial / \partial \tau .
$$

Dimensionless functions (scalar, vector and tensor), we introduce in the following way:

$$
Z_{*}=Z / Z_{1}
$$

where $Z=\left\{q, \rho, T, W, Q, \mathbf{H}, \mathbf{B}, \mathbf{D}, \mathbf{E}, \mathbf{F}, \mathbf{v}, \mathbf{u}, \mathbf{J}, \mathbf{J}_{Q}, \mathbf{J}_{c}, \hat{\boldsymbol{\sigma}}, \hat{\mathbf{e}}, \hat{\boldsymbol{\pi}}\right\}$. All values $Z_{1}$, except density $\rho$ ( $\rho_{0}$ is initial density of the body) we normalize on concentration $c_{1}$ :

$$
\begin{aligned}
t_{1} & =l^{2} / D, \quad e_{1}=\beta_{1} c_{1}, \quad u_{1}=e_{1} l, \quad v_{1}=e_{1} D / l, \quad q_{1}=\beta_{1} c_{1} / \beta, \quad B_{1}=\mu \mu_{0} H_{1}, \quad D_{1}=\varepsilon \varepsilon_{0} E_{1}, \\
J_{1} & =\lambda E_{1}, \quad J_{1 c}=\rho_{0} D c_{1} / l, \quad s_{1}=d_{1} c_{1}, \quad T_{1}=T_{c} c_{1}, \quad E_{1}=u_{0} c_{1} / l, \quad H_{1}=\varepsilon \varepsilon_{0} D u_{0} c_{1} / l^{2}, \\
Q_{1} & =\rho_{0} D \mu_{c} c_{1} / l^{2}, \quad \sigma_{1}=E_{p} e_{1} /\left(1-2 \nu_{p}\right), \quad J_{1 Q}=2 \kappa T_{0} c_{1} /\left(3 c_{n} l\right) .
\end{aligned}
$$

Here $\varepsilon_{\lambda}=\lambda \mu \mu_{0} D, u_{0}=2 \varepsilon_{\lambda} \varphi_{1} /\left(3 c_{n}\right), T_{c}=\rho_{0} D \mu / \kappa$ is characteristic temperature of diffusion process.

Given the range of definitions (13)-(15), we rewrite the system of equations of magnetothermomechanodiffusion (2)-(12) in a dimensionless values:

- Maxwell equations (2):

$$
\begin{gathered}
\left(\frac{l_{D}}{l}\right)^{2}\left(\nabla_{*} \times \mathbf{H}_{*}-\frac{\partial \mathbf{D}_{*}}{\partial \tau}\right)=\mathbf{j}_{*}, \quad \nabla_{*} \times \mathbf{E}_{*}=-\left(\frac{l_{c}}{l}\right)^{2} \frac{\partial \mathbf{H}_{*}}{\partial \tau}, \quad \nabla_{*} \cdot \mathbf{B}_{*}=0 \\
\left(\frac{l_{D}}{l}\right)^{2} \nabla_{*} \cdot \mathbf{D}_{*}=\rho_{*} q_{*}, \quad \varepsilon_{q} \rho_{*} \frac{d q_{*}}{d \tau}+\nabla_{*} \cdot \mathbf{J}_{*}=0, \quad \mathbf{J}_{*}=\mathbf{j}_{*}-e_{1} \rho_{*} q_{*} \mathbf{V}_{*}
\end{gathered}
$$

- material relations of macroscopic electrodynamics (3):

$$
\mathbf{B}_{*}=\mathbf{B}_{*}\left(\mathbf{H}_{*}-e_{1} \mathbf{v}_{*} \times \mathbf{E}_{*}\right), \quad \mathbf{D}_{*}=\mathbf{D}_{*}\left(\mathbf{E}_{*}+e_{1}\left(l_{c} / l\right)^{2} \mathbf{v}_{*} \times \mathbf{B}_{*}\right) ;
$$

- balance relations (4):

$$
\begin{gathered}
\frac{d \rho_{*}}{d \tau}+e_{1} \rho_{*} \nabla_{*} \cdot \mathbf{v}_{*}=0, \quad \rho_{*} \frac{d c_{*}}{d \tau}+\nabla_{*} \cdot \mathbf{J}_{* c}=0, \\
\hat{\mathbf{e}}_{*}=\left(\nabla_{*} \mathbf{u}_{*}+\mathbf{u}_{*} \nabla_{*}+e_{1} \nabla_{*} \mathbf{u}_{*} \cdot \mathbf{u}_{*} \nabla_{*}\right) / 2, \quad \frac{d}{d \tau}=\frac{\partial}{\partial \tau}+e_{1} \mathbf{v}_{*} \cdot \nabla_{*} ;
\end{gathered}
$$

Mathematical Modeling and Computing, Vol.2, No. 1, pp. 99-106 (2015) 


$$
\left(\frac{l_{\rho}}{l}\right)^{2} \rho_{*} \frac{d \mathbf{v}_{*}}{d \tau}=\nabla_{*} \cdot \hat{\boldsymbol{\sigma}}_{*}+\varepsilon_{F} c_{1} \rho_{*} q_{*} \mathbf{E}_{*}+\left(\frac{l_{F}}{l}\right)^{2} c_{1}\left[\mathbf{j}_{*} \times \mathbf{B}_{*}+\left(\frac{l_{D}}{l}\right)^{2} \mathbf{F}_{*}\right]
$$

where

$$
\mathbf{F}_{*}=\left[\frac{B_{*}\left(H_{*}\right)}{H_{*}}-\mu_{*}\left(H_{*}\right)\right] \nabla_{*}\left(H_{*}^{2}\right)
$$

-expressions for thermodynamic flows (6)-(7):

$$
\begin{gathered}
\mathbf{J}_{* Q}=-\varepsilon_{J T} \nabla_{*} T_{*}+\varepsilon_{\lambda}\left[\mathbf{E}_{*}+e_{1}\left(\frac{l_{c}}{l}\right)^{2} \mathbf{v}_{*} \times \mathbf{B}_{*}\right]-\nabla_{*} c_{*}+\varepsilon_{J \sigma} \nabla_{*} \sigma+\varepsilon_{J q} \nabla_{*} q_{*}, \\
\mathbf{J}_{* c}=-\nabla_{*} c_{*}-\frac{\varepsilon_{c T} c_{1} c_{*}}{2\left(1+\varepsilon_{c T} c_{1} T_{*}\right)} \nabla_{*} T_{*}+\varepsilon_{c \sigma} \nabla_{*} \sigma_{*}-\varepsilon_{c q} \nabla_{*} q_{*}, \quad \mathbf{J}_{*}=\mathbf{E}_{* s} ;
\end{gathered}
$$

- expression (8) for $E_{* s}$ :

$$
\mathbf{E}_{* s}=\mathbf{E}_{*}+e_{1}\left(l_{c} / l\right)^{2} \mathbf{v}_{*} \times \mathbf{B}_{*}-\varepsilon_{\lambda}^{-1}\left[\nabla_{*} c_{*}-\varepsilon_{j T} \nabla_{*} T-\varepsilon_{J \sigma} \nabla_{*} \sigma_{*}\right] ;
$$

- generalized Hooke's law (9):

$$
\hat{\boldsymbol{\sigma}}_{*}=\left[\frac{\nu_{p}}{1+\nu_{p}} \mathbf{e}_{*}-\varepsilon_{\sigma T} T_{*}+\frac{c_{*}}{3}\right] \hat{\mathbf{I}}+\frac{1-2 \nu_{p}}{1+\nu_{p}}\left(\hat{\mathbf{e}}_{*}-\hat{\boldsymbol{\pi}}_{*}\right) ;
$$

- heat conduction equation (10):

$$
\varepsilon_{D} \rho_{*} \frac{d T_{*}}{d \tau}=\Delta_{*} T_{*}+Q_{*}-\varepsilon_{T s}\left(1+\varepsilon_{c T} c_{1} T_{*}\right) \frac{d s_{*}^{\prime}}{d \tau}
$$

- total heat releases (11):

$$
Q_{*}=-\varepsilon_{Q Q} \nabla_{*} \cdot \mathbf{J}_{* Q}^{\prime}+c_{1}\left[\varepsilon_{Q E} E_{* s}^{2}-e_{1}\left(l_{v} l_{c} / l^{2}\right)^{2} \mathbf{v}_{*} \times \mathbf{F}_{*}-\mathbf{J}_{* c} \cdot \nabla_{*}\left(c_{*}-\varepsilon_{Q T} T_{*}-\varepsilon_{c \sigma} \sigma_{*}\right)\right] ;
$$

- expressions (12) for $s^{\prime}$ and $J_{Q}^{\prime}$ :

$$
s_{*}^{\prime}=c_{*}+\varepsilon_{s \sigma} \sigma_{*}+\varepsilon_{s q} q_{*}, \quad \mathbf{J}_{* Q}^{\prime}=\mathbf{J}_{* Q}+\nabla_{*} T_{*} / \varepsilon_{Q Q} .
$$

In equations (16)-(24) the following notation are introduced:

$$
\begin{gathered}
l_{D}=\sqrt{\frac{\varepsilon \varepsilon_{0} D}{\lambda}}, \quad l_{c}=\sqrt{\varepsilon \mu} \frac{D}{c_{0}}, \quad l_{q}=\sqrt{\frac{\varepsilon \varepsilon_{0} \beta u_{0}}{\rho_{0} \beta_{1}}}, \\
l_{\rho}=D \sqrt{\frac{\left(1-2 \nu_{p}\right) \rho_{0}}{E_{p}}}, \quad l_{F}=u_{0} \sqrt{\frac{\left(1-2 \nu_{p}\right) \varepsilon_{\lambda} \varepsilon \varepsilon_{0}}{E_{p} \beta_{1}}}, \quad l_{v}=u_{0} \sqrt{\frac{\varepsilon \varepsilon_{0}}{\rho_{0} \mu_{c}}} \\
\varepsilon_{J q}=\frac{3 \kappa_{l} \mu_{q} \beta_{1} c_{n}}{2 \kappa T_{0} \beta}, \quad \varepsilon_{q}=\frac{\rho_{0} D \beta_{1}}{\lambda \beta u_{0}}, \quad \varepsilon_{F}=\frac{\left(1-2 \nu_{p}\right) \rho_{0} u_{0}}{\beta E_{p}}, \quad \varepsilon_{c \sigma}=\frac{\mu_{\sigma} E_{p} \beta_{1}}{\left(1-2 \nu_{p}\right) \mu_{c}}, \\
\varepsilon_{c q}=\frac{\mu_{q} \beta_{1}}{\mu_{c} \beta}, \quad \varepsilon_{J T}=\frac{3 c_{n} T_{c}}{2 T_{0}}, \quad \varepsilon_{Q Q}=\frac{2 \kappa T_{0}}{3 \rho_{0} D \mu_{c} c_{n}}, \quad \varepsilon_{Q E}=\frac{\lambda u_{0}^{2}}{\rho_{0} D \mu_{c}}, \\
\varepsilon_{S \sigma}=\frac{\alpha_{T} E_{p} \beta_{1}}{\left(1-2 \nu_{p}\right) \rho_{0} d_{1}}, \quad \varepsilon_{s q}=\frac{\gamma \beta_{1}}{\beta d_{1}}, \quad \varepsilon_{D}=D / a, \quad \varepsilon_{C T}=T_{C} / T_{0}, \\
\varepsilon_{J \sigma}=\beta_{1} c_{n}, \quad \varepsilon_{j T}=3 \alpha_{T} c_{n} T_{c}, \quad \varepsilon_{Q T}=\mu_{T} T_{c} / \mu_{c}, \quad \varepsilon_{\sigma T}=\alpha_{T} T_{c} / \beta_{1}, \quad \varepsilon_{T S}=T_{0} d_{1} \mu_{c} .
\end{gathered}
$$


We perform quantitative analysis of specific criteria (25) for the case of hydrogen diffusion in iron for three values of temperature. Characteristics of the material and diffusant we take as [13-16]:

$$
\begin{array}{cl}
\varepsilon=1, \quad M_{1}=1.00794 \cdot 10^{-3} \mathrm{~kg} / \mathrm{mol}, & M_{2}=55.847 \cdot 10^{-3} \mathrm{~kg} / \mathrm{mol}, \\
c_{1 \sigma}=20.6213 \cdot 10^{3} \mathrm{~J} /(\mathrm{kg} \cdot \mathrm{K}), \quad \sigma_{n p}=1.2 \cdot 10^{8} \mathrm{~N} / \mathrm{m}^{2},
\end{array}
$$

the rest of characteristics whose values depend on the temperature are given in Table 1.

Table 1.

\begin{tabular}{|c|c|c|c|c|c|}
\hline$T_{0}, \mathrm{~K}$ & $B_{s}, \mathrm{~T}$ & $\mu$ & $\alpha_{T}, 10^{5} \mathrm{~K}^{-1}$ & $\kappa, \mathrm{V} /(\mathrm{m} \times \mathrm{K})$ & $\rho_{0}, 10^{3} \mathrm{~kg} / \mathrm{m}^{3}$ \\
\hline 300 & 2.07 & 250 & 1.22 & 74 & 7.87 \\
\hline 600 & 1.77 & 197 & 1.55 & 55 & 7.77 \\
\hline 900 & 0.92 & 28 & 1.60 & 37 & 7.66 \\
\hline \hline$T_{0}, \mathrm{~K}$ & $c_{2 \sigma}, 10^{3} \mathrm{~J} /(\mathrm{kg} \cdot \mathrm{K})$ & $\lambda, 10^{7} \mathrm{~A} /(\mathrm{V} \times \mathrm{m})$ & $E_{p}, 10^{11} \mathrm{~N} / \mathrm{m}^{2}$ & $\nu_{p}$ & $\mathrm{D}, 10^{-11} \mathrm{~m}^{2} / \mathrm{s}$ \\
\hline 300 & 0.46 & 1.02 & 2.10 & 0.28 & 1.0 \\
\hline 600 & 0.57 & 0.35 & 1.94 & 0.25 & 2.8 \\
\hline 900 & 0.68 & 0.21 & 1.78 & 0.23 & 5.2 \\
\hline
\end{tabular}

For the examine case the values of specific criteria (25), shown in Table 2, are calculated.

\begin{tabular}{|c|c|c|c|c|c|c|c|c|c|c|}
\hline$T_{0}, \mathrm{~K}$ & $l_{D}, 10^{-15} \mathrm{~m}$ & \multicolumn{2}{|c|}{$l_{c}, 10^{-19} \mathrm{~m}$} & \multicolumn{2}{|c|}{$l_{q}, 10^{-13} \mathrm{~m}$} & \multicolumn{2}{|c|}{$l_{\rho}, 10^{-15} \mathrm{~m}$} & \multicolumn{2}{|c|}{$l_{F}, 10^{-20} \mathrm{~m}$} & $l_{v}, 10^{-18} \mathrm{~m}$ \\
\hline 300 & 2.9463 & \multicolumn{2}{|c|}{5.2742} & \multicolumn{2}{|c|}{1.2958} & \multicolumn{2}{|c|}{1.2841} & \multicolumn{2}{|c|}{2.0945} & 1.7391 \\
\hline 600 & 8.4162 & \multicolumn{2}{|c|}{14.7676} & \multicolumn{2}{|c|}{0.8253} & \multicolumn{2}{|c|}{3.9626} & \multicolumn{2}{|c|}{1.0405} & 1.2266 \\
\hline 900 & 14.8068 & \multicolumn{2}{|c|}{27.4256} & \multicolumn{2}{|c|}{0.2717} & \multicolumn{2}{|c|}{7.9271} & \multicolumn{2}{|c|}{0.1758} & 0.1755 \\
\hline$T_{0}, \mathrm{~K}$ & $u_{0}, 10^{-6} \mathrm{~V}$ & \multicolumn{2}{|c|}{$T_{c}, 10^{2} \mathrm{~K}$} & \multicolumn{2}{|c|}{$\varepsilon_{C T}, 10^{-3}$} & \multicolumn{2}{|c|}{$\varepsilon_{D}, 10^{-6}$} & \multicolumn{2}{|c|}{$\varepsilon_{J \sigma}, 10^{-3}$} & $\varepsilon_{i T}, 10^{-5}$ \\
\hline 300 & 8.1993 & \multicolumn{2}{|c|}{0.2680} & \multicolumn{2}{|c|}{0.8933} & \multicolumn{2}{|c|}{0.4892} & \multicolumn{2}{|c|}{1.6710} & 1.7999 \\
\hline 600 & 6.1697 & 1.140 & & 1.90 & & & 548 & & 5647 & 9.7113 \\
\hline 900 & 0.9694 & 2.795 & & 3.10 & & & 209 & & 7327 & 24.5177 \\
\hline$T_{0}, \mathrm{~K}$ & $\varepsilon_{\lambda}, 10^{-8}$ & $\varepsilon_{q}, 10^{-2}$ & & $10^{-7}$ & $\varepsilon_{c \sigma}$ & $10^{-3}$ & $\varepsilon_{c q}$, & & $\varepsilon_{J T}, 10^{-3}$ & $\varepsilon_{J q}, 10^{-7}$ \\
\hline 300 & 3.2044 & 0.0517 & & 565 & 1.5 & 879 & 3.97 & & 2.4589 & 0.1288 \\
\hline 600 & 2.4261 & 1.0399 & & 518 & 2.9 & 321 & 6.11 & & 5.2211 & 1.3887 \\
\hline 900 & 0.3842 & 29.6942 & & 895 & 3.8 & 164 & 6.94 & & 8.5131 & 6.1733 \\
\hline$T_{0}, \mathrm{~K}$ & $\varepsilon_{\sigma T}, 10^{-3}$ & $\varepsilon_{T S}, 10^{-3}$ & & $2 Q$, & $\varepsilon_{Q E}$, & $10^{-8}$ & $\varepsilon_{Q T}$, & $0^{-4}$ & $\varepsilon_{S \sigma}, 10^{-4}$ & $\varepsilon_{s q}, 10^{-6}$ \\
\hline 300 & 3.5906 & 1.1258 & 406 & 6777 & 34. & 763 & 1.17 & & 7.1240 & 2.1577 \\
\hline 600 & 9.0809 & 3.9254 & 191 & 5236 & 2.1 & 242 & 8.26 & & 7.9852 & 3.8726 \\
\hline 900 & 14.2561 & 7.1603 & & 5127 & 0.0 & & 23.8 & & 7.1033 & 5.5311 \\
\hline
\end{tabular}

Table 2.

The orders of the values, given in Table 2 , are from -14 to +7 . Thus, the system (16)-(24) can be simplified (condition) if we neglect the terms that contain characteristic dimensionless parameters smaller than $10^{-3}$. That is, for the case of magnetomechanothermodiffusion in the ferromagnetic body at given concentration of diffusing substance on the surface all dimensionless parameters (equation (25)) (except $\varepsilon_{E T}, \varepsilon_{\varphi}, e_{1}, \varepsilon_{c T}, \varepsilon_{J c}$ ) can always be neglected since they are much smaller than a unity. Since the real characteristic macroscopic body sizes $l$ are always larger than the characteristic sizes $l_{D}, l_{c}$, $l_{\rho}$, we will always neglect all terms that contain these values. 
This means that in this approximation we can take: $q_{*}=0, \mathbf{j}_{*}=\mathbf{J}_{*}=0$, and, therefore $\mathbf{H}_{0}=0$, $\mathbf{B}_{*}=0, \mathbf{E}_{*}=0, \mathbf{D}_{*}=0$.

Also we neglect the parameter $e_{1}=\beta_{1} c_{1}$, since for concentrations $c_{1}<1 \%$ we obtain $e_{1} \approx 9 \cdot 10^{-4}$. Then from equations (18) follows that $\rho_{*}=1\left(\rho=\rho_{0}\right)$, i.e. the density of the mass distribution can be considered constant always. Disappears the difference between the substantial and local derivatives, that is $d / d t=\partial / \partial t$. The relationship between the strain tensor and displacement vector we take linear. In the heat conduction equation (23) the parameter $\varepsilon_{D}$ is neglected (dynamics of the process of heat conduction in this case is several orders of magnitude faster than diffusion).

If you disregard the above-mentioned criteria, the initial system of equations of magnetomechanothermodiffusion (2)-(12) will be of simplified form.

Write down it:

- balance relation (4):

$$
\rho \frac{\partial c}{\partial t}+\nabla \cdot \mathbf{J}_{c}=0, \quad \hat{e}=(\nabla \mathbf{u}+\mathbf{u} \nabla) / 2, \quad \nabla \cdot \hat{\sigma}=0
$$

- expressions (6)-(7) for the flows

$$
\begin{aligned}
& \mathbf{J}_{Q}=-\frac{2 \kappa T_{0}}{3 c_{n}}\left(\nabla c-\frac{1-2 \nu_{p}}{E_{p}} c_{n} \nabla \sigma\right), \\
& \mathbf{J}_{c}=-\rho_{0} D\left(\nabla c+\frac{c}{2 T} \nabla T-\frac{\mu_{\sigma}}{\mu_{c}} \nabla \sigma\right) ;
\end{aligned}
$$

- expression (8) for:

$$
\mathbf{E}_{s}=-\frac{2 \varphi_{1}}{3 c_{n}}\left(\nabla c-3 c_{n} \alpha_{T} \nabla T-\frac{1-2 \nu_{p}}{E_{p}} c_{n} \nabla \sigma\right)
$$

- heat conduction equation (10):

$$
\Delta T+\frac{1}{\kappa}\left[Q-\rho_{0} d_{1} T \frac{\partial c}{\partial t}\right]=0
$$

where

$$
Q=\frac{2 \kappa T_{0}}{3 c_{n}} \Delta\left[c-\frac{1-2 \nu_{p}}{E_{p}} c_{n} \sigma\right]-\mu_{c} \mathbf{J}_{c} \cdot \nabla\left(c-\frac{\mu_{\sigma}}{\mu_{c}} \sigma\right) .
$$

Note that the generalized Hooke's law (9) in this approximation remains unchanged.

\section{A simplified mathematical model of mechanothermodiffusion}

A simplified system of equations (26)-(30) was obtained under the condition when the parameters $\varepsilon_{i}$ were neglected ( $i$ a set of lower indices of parameters in Table 2 ) and the terms standing next to them as well, if the dimensionless parameters are one ten thousand part of a unit. Note that on the parameters $\varepsilon_{i}$ can be imposed more strict conditions. For example, if you reject those characteristic parameters $\varepsilon_{i}$ and terms standing next to them which make less than one percent compared to the unit $\left(\varepsilon_{i}<10^{-2}\right)$, you can obtain even more simplified system of equations. In this case the system of equations (26)-(30) can be written in a closed form for the concentration and temperature

$$
\begin{aligned}
& \frac{\partial c}{\partial t}=D \nabla \cdot\left(\nabla c+\frac{c}{2 T} \nabla T\right), \\
& \Delta T+T_{c}\left(\nabla c+\frac{c}{2 T} \nabla T\right) \cdot \nabla c+\frac{2 T_{0}}{3 c_{n}} \Delta c=0,
\end{aligned}
$$

Mathematical Modeling and Computing, Vol.2, No. 1, pp. 99-106 (2015) 
and the influence of diffusion process on the mechanical stresses to consider by Hooke's law

$$
\hat{\sigma}=\frac{E_{p}}{1-2 \nu_{p}}\left[\frac{\nu_{p}}{1+\nu_{p}} e+\frac{\beta_{1}}{3} c\right] \hat{I}+\frac{E_{p}}{1+\nu_{p}} \hat{e}-\hat{\pi} .
$$

\section{Conclusions}

Thus, the process of mechanothermodiffusion in ferromagnetic bodies at a given concentration of diffusing substance on the surface depends only on thermal, diffusion and atomic characteristics of diffusant and skeleton and practically does not depend neither on magnetic for electric properties of the material.

The system of equations (31), (32) can serve as a working system of equations in describing the thermal diffusion phenomena, which are used in industrial processes of applying different types of coatings on steel parts of machines and mechanisms.

Further research is necessary for magnetomechanothermodiffusion processes in ferromagnetic bodies under simultaneous action of two external factors: the time periodic electromagnetic field and given concentration of diffusant on the surface.

[1] Solodyak M. T. Thermodynamic potentials for multicomponent solid solutions, part I. Math. Methods and Phys.-Mech. Fields. 43, n.1, 173-179 (2000).

[2] Solodyak M. T. Thermomechanodiffusive processes in nonelectroconductive bodies in the external periodic magnetic field. Phys.-Chem. Mechanics of Materials. 36, n.6, 29-32 (2000).

[3] Groot S. P. de, Mazur P. Non-equilibrium thermodynamics. Mir, Moscow (1964).

[4] Solodyak M. T, Ivas'ko R. O. Minkowski equations in nonlinear magnetizable and polarizable moving media. Math. Methods and Phys.-Mech. Fields. 42, n.1, 50-54 (1999).

[5] Solodyak M. T. Mathematical model of magnetothermoelasticity of ferromagnetic bodies. Phys.-Chem. Mechanics of Materials. 36, n.1, 7-16 (2000).

[6] Tables of physical quantities: Reference book / Ed. Kikoin I. K. Atomizdat, Moscow (1976).

[7] Solodyak M. T. Thermodynamic potentials for multicomponent solid solutions, part II. Math. Methods and Phys.-Mech. Fields. 43, n.4, 140-145 (2000).

[8] Solodyak M. T. About covariance of ponderomotive force and energy-momentum tensor in continuum mechanics. Math. Methods and Phys.-Mech. Fields. 42, n.2, 147-154 (1999).

[9] Bazarov I. P., Gevorgyan E. V., Nikolaev P. N. Non-equilibrium thermodynamics and physical kinetics. MGU, Moscow (1989).

[10] Kaye G. W. C., Laby T. H. Tables of physical and chemical constants and some mathematical functions. Nabu Press (2010).

[11] Kolesnikov P. M. Introduction to nonlinear electrodynamics. Nauka i tiekhnika, Minsk (1971).

[12] Livshits B. G., Kramoshin V.S., Linetskiy J.L. Physical properties of metals and alloys. Metallurgia, Moscow (1980).

[13] Sedov L. I. Similarity and dimensional methods in mechanics. Nauka, Moscow (1977).

[14] Belov K. P. Elastic, thermal and electrical phenomena in ferromagnetic metals. Gostekhizdat, Moscow (1951).

[15] Filippov T. Many faces of soliton. Nauka, Moscow (1986).

[16] Solodyak M.T. Thermomechanodiffusive processes in electroconductive bodies in the external time periodic magnetic field. Phys.-Chem. Mechanics of Materials. 36, n.5, 91-98 (2000). 


\title{
Дослідження магнітомеханотермодифузійних процесів у феромагнітному тілі за заданої на поверхні концентрації дифундуючої речовини
}

\author{
Солодяк М. I.
}

Інститу прикладних проблем механіки і математики ім. Я. С. Підстригача НАН України вул. Наукова, 3б, 79060, Львів, Україна

\begin{abstract}
Запропоновано вихідні рівняння, що описують процеси взаємодії електромагнітного поля з речовиною. 3 використанням методів теорії подібності та розмірностей систему рівнянь магнітомеханотермодифузї зведено до безрозмірної форми. Виконано кількісний аналіз характерних критеріїв у випадку дифузї водню в залізо для трьох значень початкової температури. У вихідній моделі нехтується ефектами, для яких характерні безрозмірні параметри є набагато меншими від одиниці. Записано спрощену взаємозв'язану систему рівнянь моделі для знаходження концентрації та температури.
\end{abstract}

Ключові слова: електромагнітне поле, магнітомеханотермодифузіл, феромагнітне тіло, концентрачіл дифундуючої речовини, методи теорї подібності та розмірностей

2000 MSC: $58 \mathrm{~J} 65$

Удк: 539.3:537.22 